\title{
FPGA Based Low Cost Automatic Test Equipment for Digital Circuits
}

\author{
Alp Arslan Bayrakci \\ Department of Computer Engineering, Gebze Technical University, Kocaeli, Turkey
}

Cite this article as: Bayrakci AA. FPGA Based Low Cost Automatic Test Equipment for Digital Circuits. Electrica, 2019; 19(1): 12-21.

\section{ABSTRACT}

Testing of digital circuits is a crucial problem. There are two types of Automatic Test Equipment (ATE): Very precise but complex and expensive test equipments called high-end ATE and their approximate but cheap alternatives called low-end ATE. In this paper we propose a very cheap, FPGA based embedded low-cost ATE (ELATE) that is capable of functional, speed/delay and power consumption tests. It is composed of FPGA hardware with six FSM modules written in Verilog and a computer software (user interface) communicating with the FPGA through UART. It can handle different I/O combinations and can detect delay with $4 \mathrm{~ns}$ precision. It can both visually show the resultant voltage/current-time graphs and store them as text files. The ATE is tested on different Design Under Test (DUT) devices like 8-bit and 12-bit adders and a square root circuit implemented on FPGA.

Keywords: FPGA, automatic test equipment, digital circuit, functional, delay, power, test

\section{Corresponding Author:}

Alp Arslan Bayrakci

E-mail:

abayrakci@gtu.edu.tr

Received: 30.04 .2018

Accepted: 30.07 .2018

(c) Copyright 2019 by Electrica

Available online at

http://electrica.istanbul.edu.tr

DOI: 10.26650/electrica.2018.28093

\section{Introduction}

Importance of digital circuit testing increases while the complexity and difficulty to test also increase. There are different approaches in the literature for testing of digital circuits. Tests of ASICs with hundreds of input output pins and speeds at $\mathrm{GHz}$ rates are really complex and difficult. On the other hand many types of digital circuit prototypes need to be tested much before manufacturing phase.

This necessitates two types of automatic test equipments (ATE): high-end and low-end. Highend ATE is powerful, fast but expensive and complex. Big companies such as Advantest [1] and Teradyne [2] construct such fast and complex ATE with multi million dollars of cost in order to test many manufactured ASICs in a small amount of time and for many input test vectors. However, they are difficult to learn and too costly to be used by many digital circuit designers.

On the other hand, low-end ATE is relatively cheap and easier to use. There are different companies like Teseda [3], which try to lower the ATE cost [4]. Recently, due to the improvements at the FPGA technology, ATE based on FPGAs are popular in the literature [5, 6, 7]. They either can only handle functional tests or require reconfigurability to be adapted to different test circuits.

In this paper, we devise an ATE, capable of performing functional, speed and power tests without the need for reconfiguration, adaptively detecting when to stop the tests and consuming a reasonable amount of space. It was first introduced in [8] roughly. In this paper, we not only present the technical implementation of ATE using software and FSMs missing in [8] but also perform more tests on different arithmetic circuits implemented on FPGA. Also the use of satisfiability (SAT) is suggested to support functional tests.

Section 2 proposes the use of satisfiability for functional test expected output computation. Section 3 explains the implemented ATE software and Section 4 gives the technical implementation of ATE hardware module by module to enable researchers implement their own ATE. Last section presents the results. 


\section{Satisfiability for Functional Tests}

Satisfiability can be used to detect the output of the circuit when a particular input is given as well as detecting true paths in the circuit.

The circuit description can be converted into a conjunctive normal form (CNF) formula. Each logic gate has its own CNF convertion shown in as shown in Table 1 [9]. After converting each logic gate in the circuit into the equivalent CNF form and ANDing these CNFs into one CNF representing the whole circuit, the input test vector for which the corresponding output is required, must be inserted into this circuit CNF.

Table 1. Conversion of logic gates to 3-CNF

\begin{tabular}{ll}
\hline Logic Gate & CNF \\
\hline$F=A^{\prime}(N O T)$ & $(A+F) \cdot\left(A^{\prime}+F^{\prime}\right)$ \\
\hline$F=A+B(O R)$ & $\left(A+B+F^{\prime}\right) \cdot\left(A^{\prime}+F\right) \cdot\left(B^{\prime}+F\right)$ \\
\hline$F=A \cdot B(A N D)$ & $\left(A^{\prime}+B^{\prime}+F\right) \cdot\left(A+F^{\prime}\right) \cdot\left(B+F^{\prime}\right)$ \\
\hline
\end{tabular}

*All other gates can be derived from AND, OR and NOT
The resultant circuit CNF can be used to detect the expected output when the primary inputs are inserted into the CNF circuit description. For that purpose, the primary input signals with logic-1 should be directly inserted to CNF whereas for others (with logic-0) the complements of the primary input signals must be inserted into the circuit CNF. The resultant CNF formula can be given to a sat-solver [10]. As a result, all logic values for all nodes inside the circuit as well as the primary outputs can be extracted from the sat-solver result. The primary output values gathered from this result are the expected outputs for the corresponding input vector, which can be used in functional tests as expected outputs that will be clarified later.

\section{ATE Software}

Automatic test equipments software is written by C\# on Visual Studio IDE. It is responsible from the communication with ATE hardware, adjustment of test vectors/settings and visualizing the test results with necessary graphics. It has to parse the incoming message from the hardware and convert it to the visual graphs and text files filled with the results. There are different tabs for settings, functional test, propagation delay test and power consumption test as shown in Figure 1.

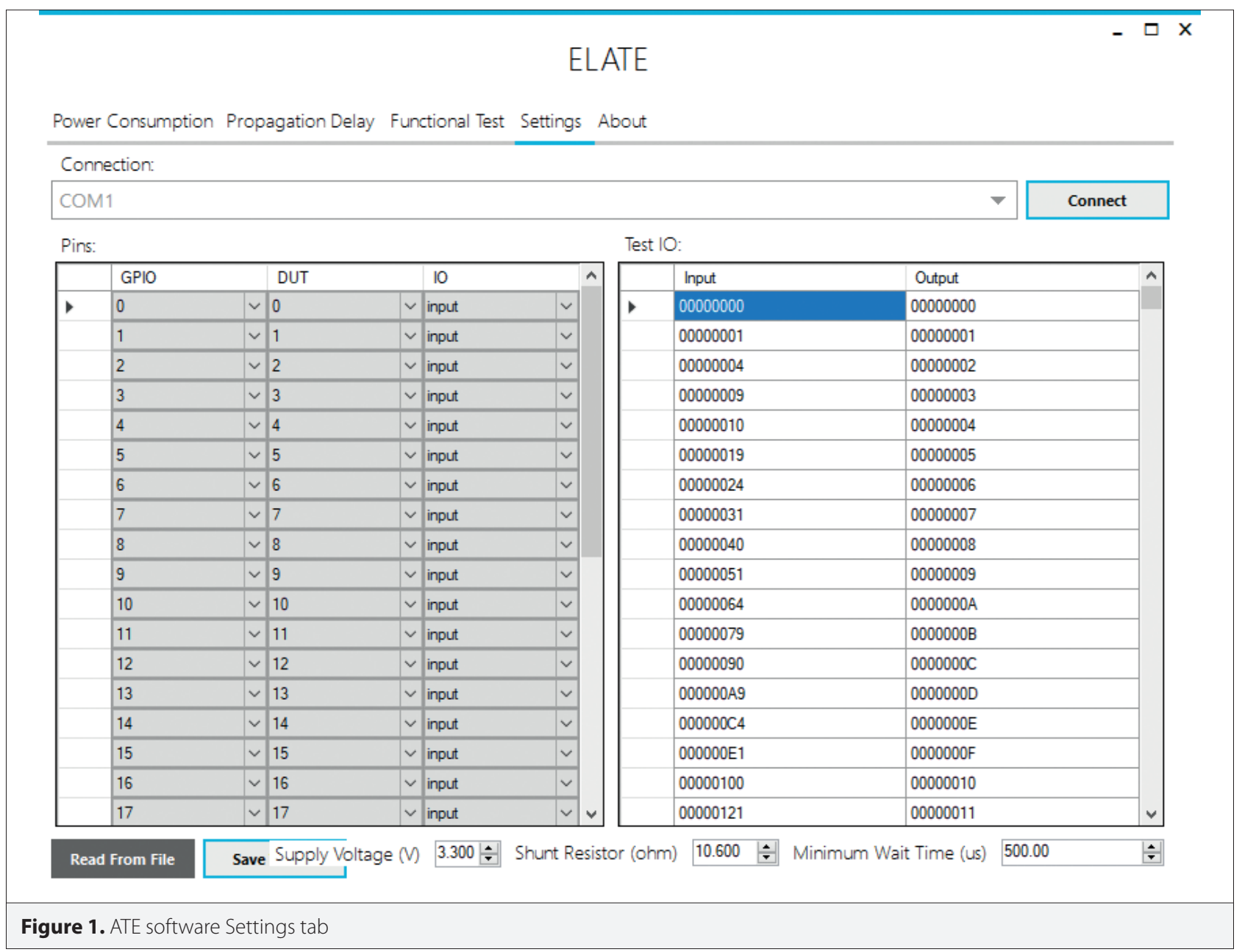


Settings tab includes four panes as shown in Figure 1. Top pane is for selecting the UART communication port with the ATE hardware. Left pane is for setting the input/output pin configurations. Right pane is for entering the input test vectors and the expected output vectors. Bottom pane is for entering the minimum wait time, supply voltage and shunt resistor values. All these settings can be saved as text file or they can be imported from a text file as shown in Figure 2 . All vectors are given in hex format. This text file can be generated automatically by an automatic test pattern generation algorithm similar to the one devised in Section 2.1.

Functional test tab explained in Section 5.1 has a start test button for starting the functional test with the vectors shown in Settings tab. Each output test vector is taken from ATE hardware through UART communication and shown at the functional test tab until all test vectors are over. If the resultant actual output vector is different than the expected one it is shown with red filled cell otherwise it is shown with green filled cell.

Propagation delay tab explained in Section 5.2 has two buttons, one for starting the tests and other for saving the results to text file. There is also a graphics pane to show output signals corresponding to an input vector.

Power consumption tab is very similar to the propagation delay tab except the graph shows time vs. supply current data instead of output voltages. The resultant graphs can also be stored as jpeg or png images.

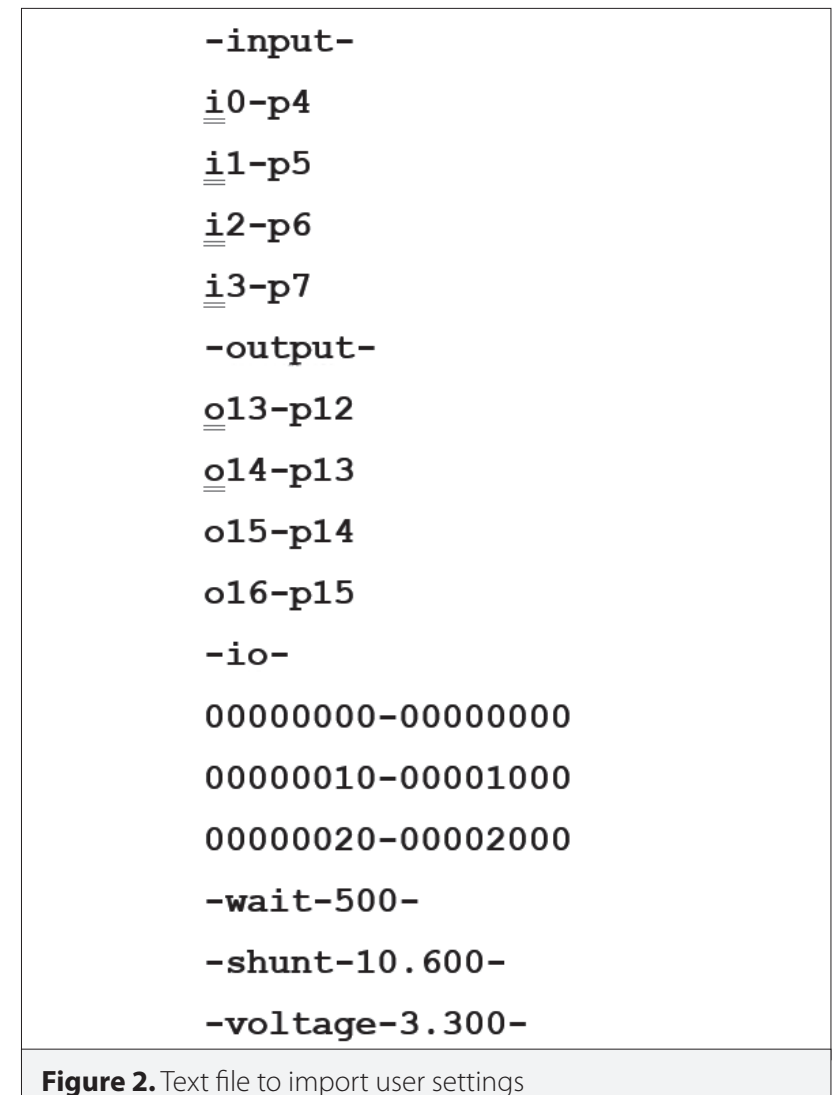

Figure 2. Text file to import user settings

\section{ATE Hardware}

Proposed ATE has six main modules each of which is designed as finite state machine (FSM) in Verilog HDL and with Quartus II v16.1 software of Altera.

\section{UART module}

UART module consists of an eight state FSM for receiving data from the ATE software and a three state FSM for transmitting data to the ATE software. It is responsible not only to receive all user settings and the input test vectors but also to send back all test results to the ATE software. It is basically the slightly modified version of the module in [11]. The UART communication is handled through a USB cable using virtual COM port drivers at the computer side. Different BAUD rates can be adjusted by setting parameters inside the module.

\section{SPI module}

This is a four state FSM module, which is only used to communicate with the analog to digital converter (ADC). It implements the SPI communication protocol and reads the resultant 12 bit digital output of the ADC chip whenever a measurement is required.

\section{Test control module}

This is one of the most important modules of ATE hardware. Having an eight state FSM, it is responsible to perform the pin

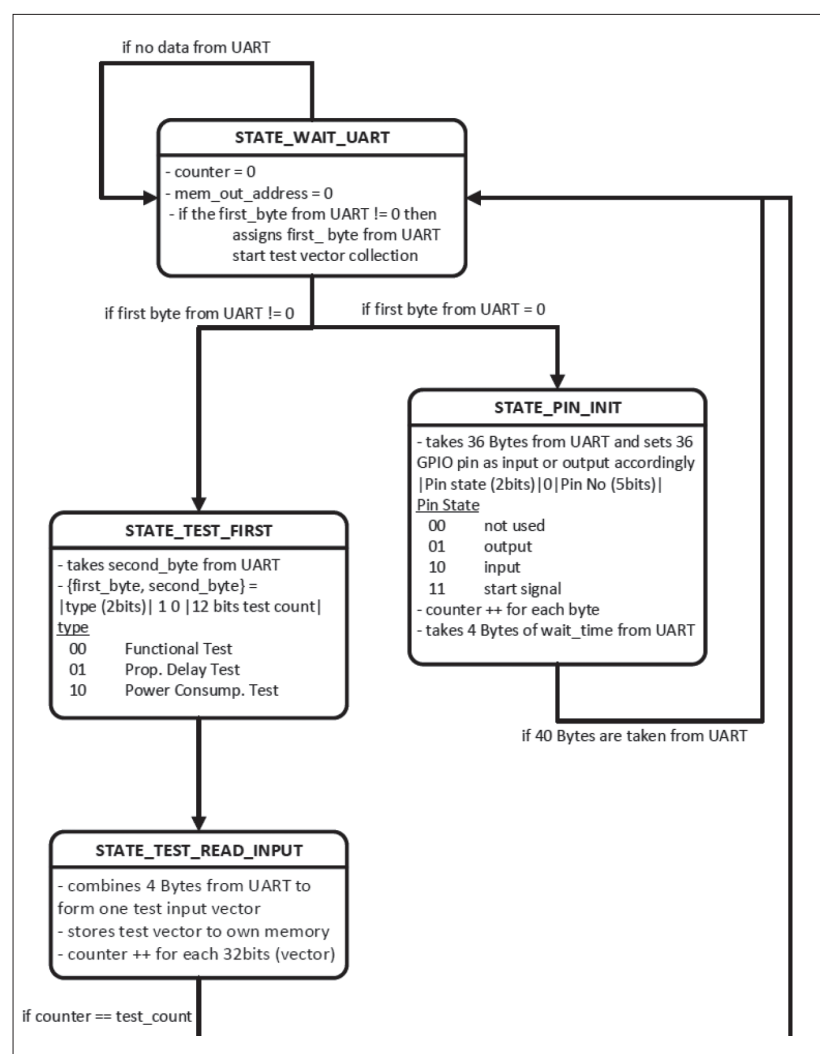

Figure 3. First four stages of Test Control Module 
input/output settings according to the user inputs, store input test vectors in its internal memory, start and manage all tests. Figure 3 shows the first four states while Figure 4 shows the second four states of the module.

STATE_WAIT_UART is the idle state waiting for incoming data from UART module. When a byte of data comes it checks whether it is zero. If it is zero, it transits into STATE_PIN_INIT state, where it adjusts each GPIO pin either as input or output

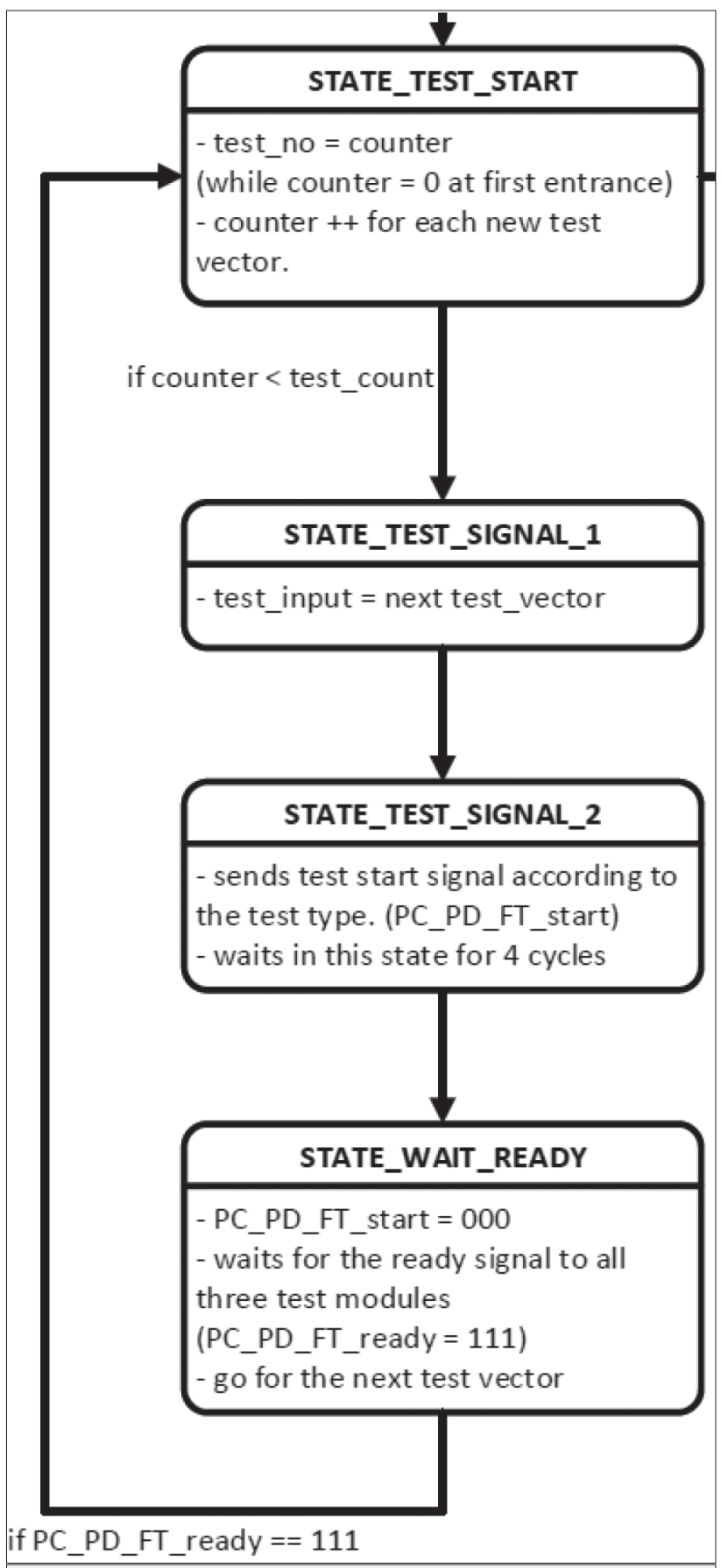

Figure 4. Second four stages of Test Control Module pin or not used. All GPIO pin settings are resolved at this state and by using the circuit shown in Figure 5.

In Figure 5 red output_select[31:0] signal and brown input_select[35:0] signal are determined according to the pin setttings coming from UART. These two signals adjusts a GPIO pin using the multiplexers shown by two column of rectangles in the figure. Left hand side multiplexor input signals are 36 GPIO pins whereas the input signals of right hand side multiplexors are 32 bits inputs. This circuit enables to adjust any GPIO pin of the ATE hardware as input to DUT or as output from DUT, which resolves the issue of compatibility to any test circuit with any input/output pin configuration provided that the total number of pins is bounded by 36 .

This solution is better than reconfiguring the ATE hardware (FPGA) each time the DUT is changed as devised in [5].

After the multiplexor select signals are adjusted according to the current DUT by STATE_PIN_INIT state and with the first incoming byte the state changes to STATE_TEST_FIRST where two bytes incoming from UART are combined and parsed in order to understand the test type (functional, delay or power). After the test type is determined in order to receive input test vectors the next state is STATE_TEST_READ_INPUT. FSM stays at this state until all input test vectors are stored inside the internal memory of Test Control Module.

Last four states shown in Figure 4 are used for testing the DUT with each input test vector. The next two states (...TEST_START and ..._SIGNAL_1) are responsible from sending the next stored input test vector to the corresponding test module. Then, STATE_TEST_SIGNAL_2 sends the start signal to the same module. This start signal activates the corresponding module that is responsible for finalizing the test and sending all results back to ATE software via UART module. STATE_WAIT_READY state waits until the testing for the corresponding test vector finishes and all results are sent back. After all tasks corresponding to the current input test vector finishes, the next test is initiated until all input test vectors are utilized and test is over.

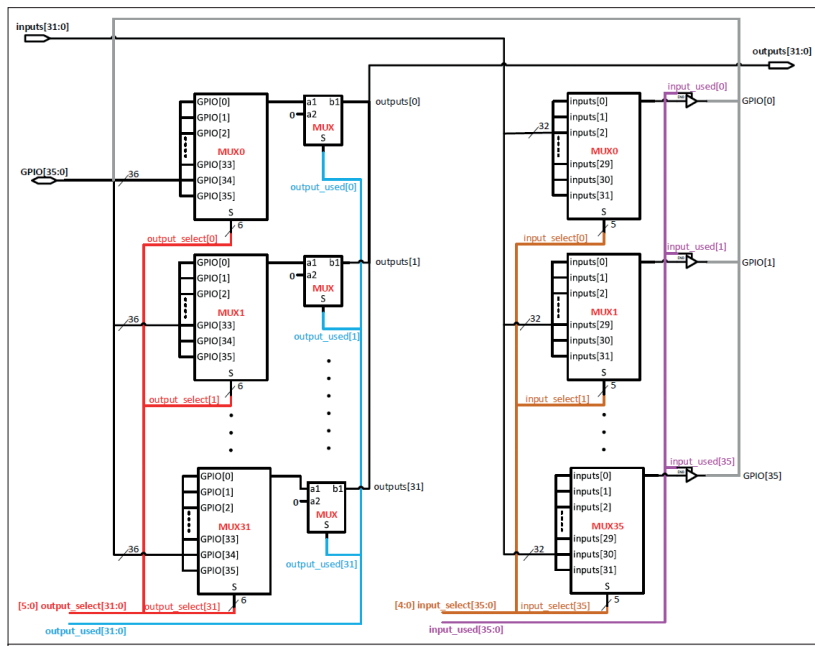

Figure 5. Pin Adjustment circuit in Test Control Module 


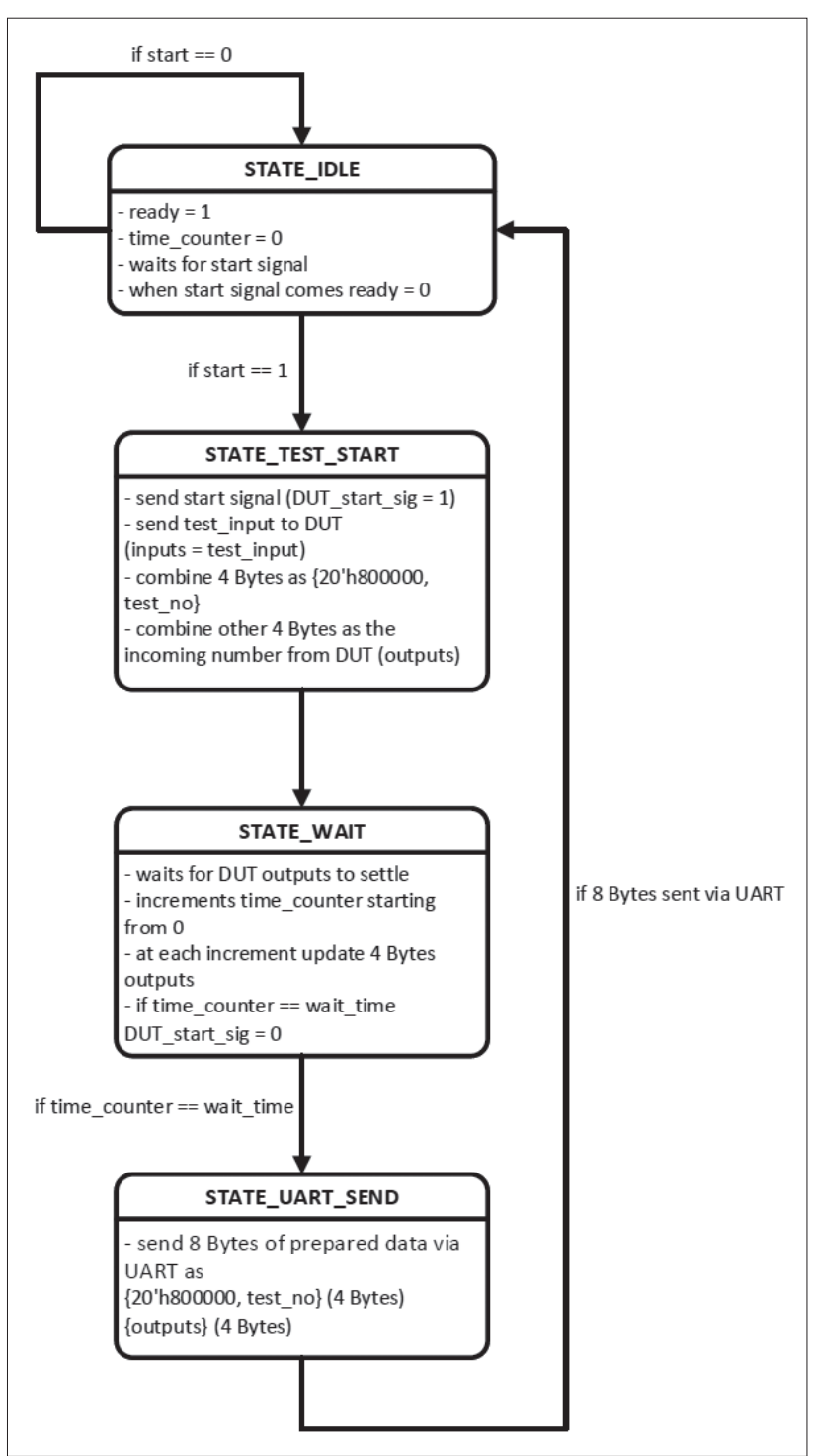

Figure 6. Functional Test Module FSM

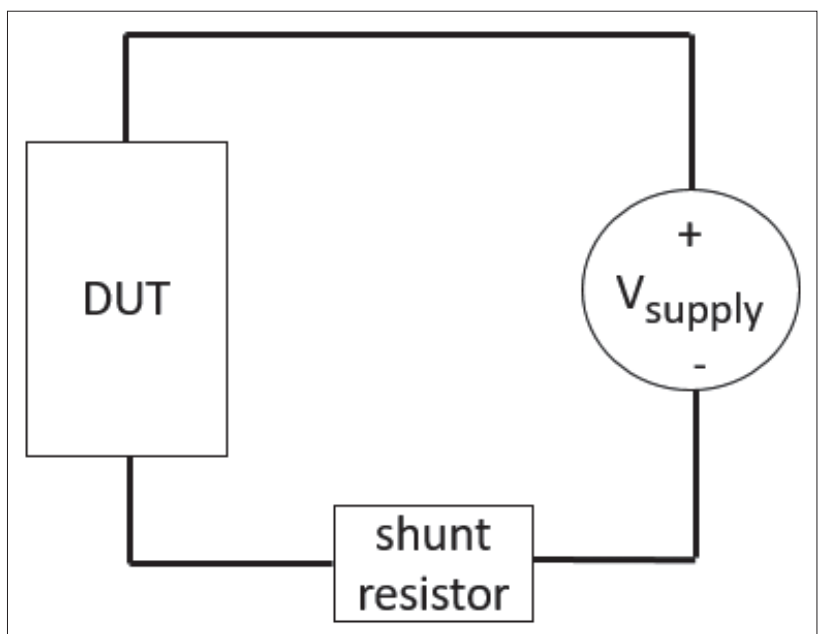

Figure 7. Connection of shunt resistor for power tests

\section{Functional test module}

This module has four states as shown in Figure 6 . It waits at STATE_IDLE until a start signal comes from Test Control Module. Start signal initiates the test by transisiton to STATE_START_ TEST state, where it connects the current test vector coming from Test Control Module to the DUT inputs. Then it changes into STATE_WAIT state where it stays until the outputs are stabilized.

This part is crucial in order to ignore glitches at any of the outputs. At this state, it waits a user given amount of time, which is specified through ATE software Settings Tab. During that time period if any of the outputs changes the timer is reset to zero. As a result, this state can only be exited when none of the outputs changes during the user specified minimum wait time. This guarantees that the outputs are stabilized. Resultant stable DUT outputs are recorded and the state changes to STATE_UART_SEND where the resultant DUT output is sent back to ATE software through UART Module. Then for the next input test vector, the iteration restarts from STATE_IDLE until all test iterations are over.

Here there is another critical choice of sending all data corresponding to a test vector back to ATE software before starting next test. This method results in much lower memory utilization than storing all data corresponding to all test vectors.

\section{Propagation delay test module}

This module has four states. It is very similar to the functional test module except it traces the time information and also records the time instant at which an alteration occurs at one of the outputs. It checks the output signals 250,000,000 times in one second, i.e. with 4 nanoseconds intervals, which means that it cannot precisely detect delays for circuits faster than $250 \mathrm{MHz}$.

The main problem of this method is that recording the output signals 250 million times in one second require a huge amount of space. Nonetheless, output alterations must be recorded in order to visually see the voltage vs. time plots of all output signals. We avoid this issue by just recording the times that an output signal changes inside the memory module. By this way, instead of 250 million records, the number of records are limited by the number of output alterations. Allowing about 32000 alterations, a 256KB memory is practically enough. Second precaution is that all data corresponding to an input test vector is sent back to ATE software before starting the new test with the next input test vector.

\section{Power consumption test module}

This module has four states similar to the functional test module. It stays at STATE_IDLE until a start signal comes. Then it transits into STATE_ADC_READ, where it starts the analog to digital conversion of the voltage over the the shunt resistor connected to the circuit as in Figure 7. The voltage values are read via SPI Module at this state. 


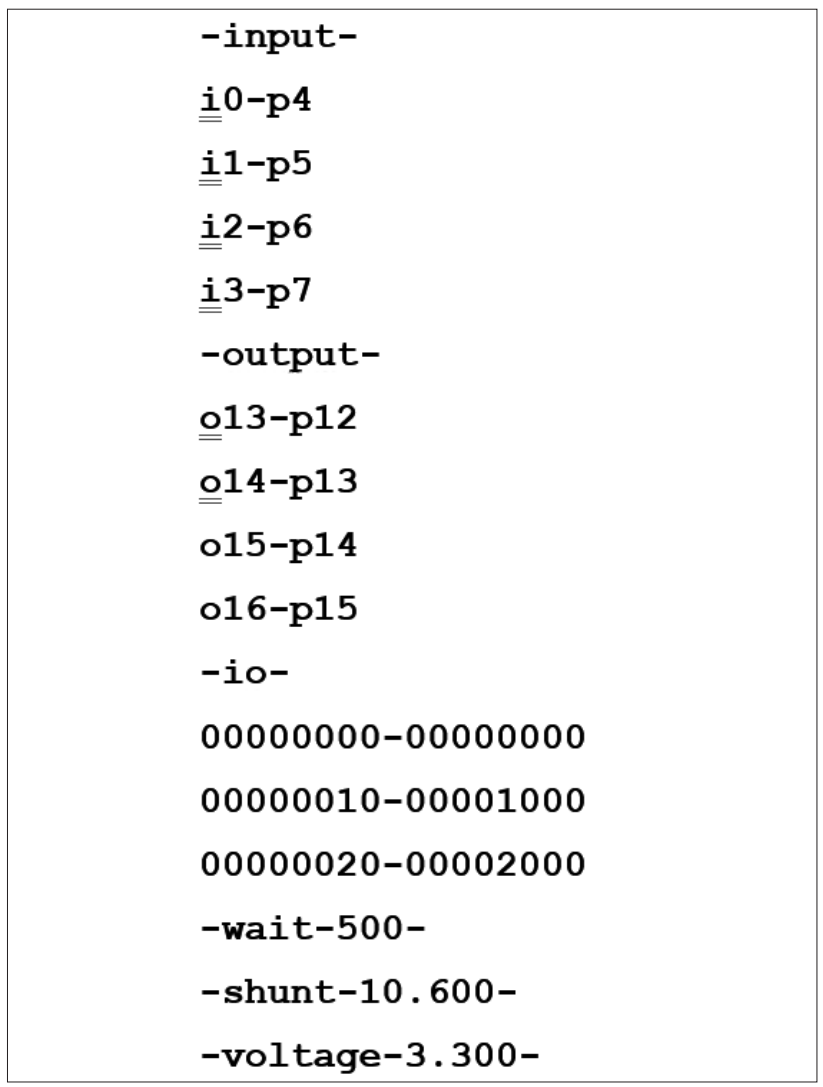

STATE_BEGIN: begin

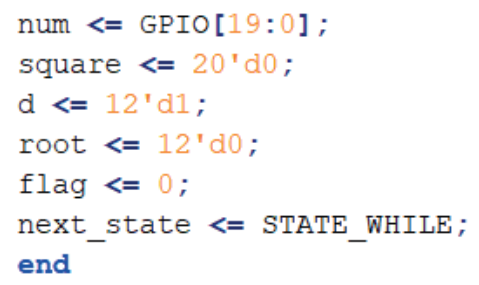

Figure 8. Integer square root implemented as FSM (root=sqrt (num))
At this point, it is important to know when to stop the ADC readings. In our solution, the reading of the analog voltage values continues until the values return back to the values at the idle state, which means that the dynamic power consumption due to the given input test vector is over.

The last state named as STATE_UART_SEND sends all recorded data of the current input test vector back to the ATE software through UART module as it is done in delay test. Then the FSM returns back to the initial state, i.e. STATE_IDLE in order to start the test for the next input test vector coming from Test Control Module.

Again the strategy to send back all data of the current test vector before switching to the next test vector avoids the memory insufficiency. Another technique that helps that is to avoid any recording unless there is a significant change in the shunt resistor voltage values, which is used to compute the instantaneous current drawn from the supply, and plotted as current vs. time graph.

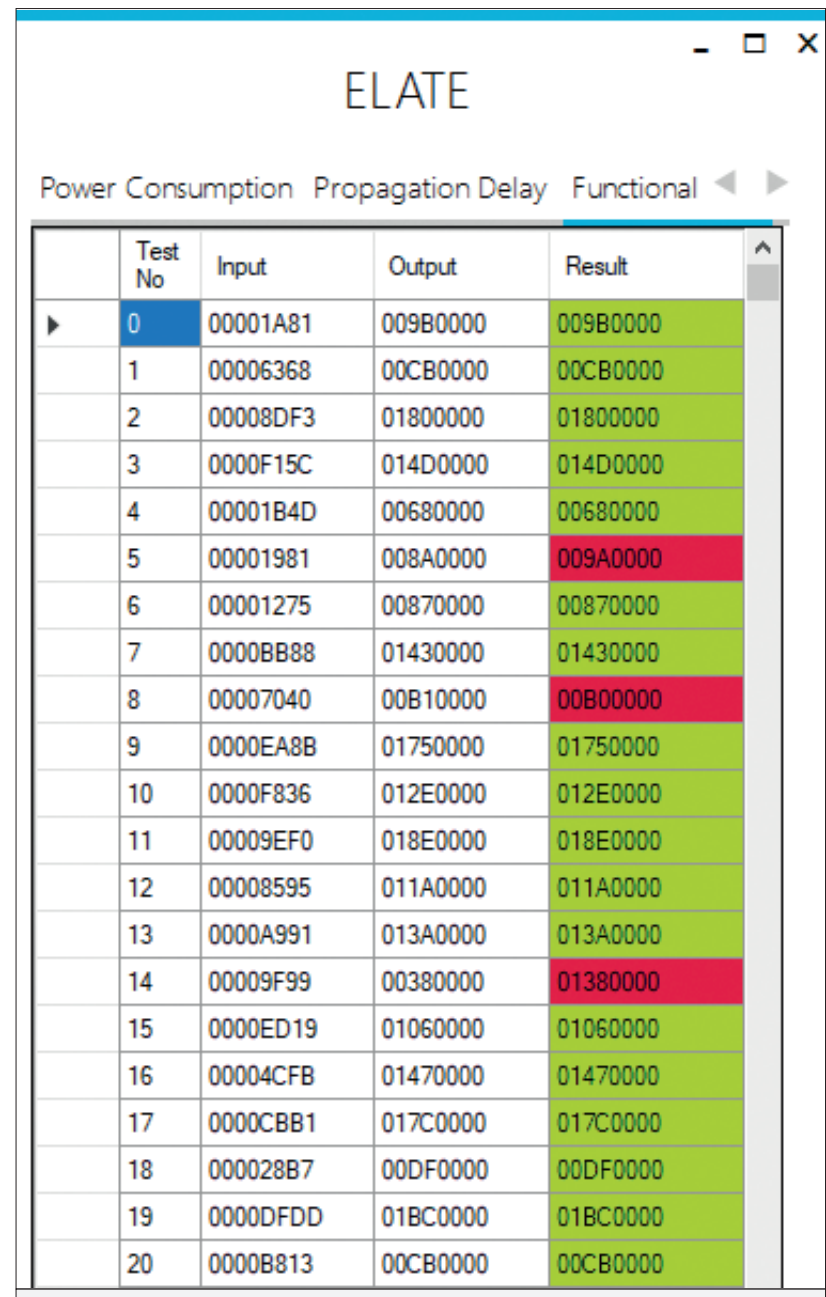

Figure 9. 8-bit adder functional test result 


\section{Results}

The test circuits are implemented on Altera Cyclone V GX board of Terasic. Tests performed for 8-bit, 12-bit adders and a square root unit written in Verilog by Quartus II tool. All test vectors are generated by a $C$ program and given as text file input to the ATE software.

The minimum wait time supplied by the user is determined adaptively by checking the functional test results and increasing the minimum wait time until all results match with the expected ones.

We implemented a square root arithmetic circuit unit on Altera Cyclone V FPGA by writing the module with two state FSM as shown in Figure 8. It takes 20 bits of input and 12 bits of output as the integer square root of the input. The adders are written in behavioral Verilog using the classical ' + ' operator.

\begin{tabular}{|c|c|c|c|c|c|c|}
\hline \multicolumn{4}{|c|}{ Power Consumption Propagation Delay } & \multicolumn{3}{|c|}{ Functional $4>$} \\
\hline & Test No & Input & Output & Result & $\hat{\wedge}$ & \\
\hline \multirow[t]{21}{*}{ 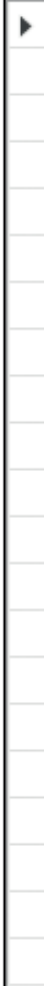 } & 0 & 00000000 & 00000000 & 00000000 & & \\
\hline & 1 & 00000001 & 00000001 & 00000001 & & \\
\hline & 2 & 00000004 & 00000002 & 00000002 & & \\
\hline & 3 & 00000009 & 00000003 & 00000003 & & \\
\hline & 4 & 00000010 & 00000004 & 00000004 & & \\
\hline & 5 & 00000019 & 00000008 & 00000005 & & \\
\hline & 6 & 00000024 & 00000006 & 00000006 & & \\
\hline & 7 & 00000031 & 00000007 & 00000007 & & \\
\hline & 8 & 00000040 & 00000008 & 00000008 & & \\
\hline & 9 & 00000051 & 00000009 & 00000009 & & \\
\hline & 10 & 00000064 & 00000003 & $0000000 \mathrm{~A}$ & & \\
\hline & 11 & 00000079 & $0000000 \mathrm{~B}$ & $0000000 \mathrm{~B}$ & & \\
\hline & 12 & 00000090 & $0000000 \mathrm{C}$ & $0000000 \mathrm{C}$ & & \\
\hline & 13 & 000000A9 & $0000000 D$ & 00000000 & & \\
\hline & 14 & $000000 C 4$ & $0000000 \mathrm{E}$ & $0000000 E$ & & \\
\hline & 15 & 000000E1 & $0000000 \mathrm{~F}$ & $0000000 \mathrm{~F}$ & & \\
\hline & 16 & 00000100 & 00000010 & 00000010 & & \\
\hline & 17 & 00000121 & 00000011 & 00000011 & & \\
\hline & 18 & 00000144 & 00000012 & 00000012 & & \\
\hline & 19 & 00000169 & 00000013 & 00000013 & & \\
\hline & 20 & 00000190 & 00000014 & 00000014 & $\checkmark$ & \\
\hline \multicolumn{6}{|c|}{ Start Test } & \\
\hline
\end{tabular}

Figure 10. Square root functional test result

\section{Functional test results}

The results of functional test for 8-bit adder and square root units are shown by Figure 9 and 10 respectively. Only the first 20 test vectors are shown for demonstration purposes. The input column shows the input vector, the output column shows the expected output and the result column shows the actual result taken from DUT. Green represents that the DUT result is same as the expected output. As it is seen in both figures there are red entries which means that the result is different from the expected output. The red entries are made intentionally in order to show that the ATE works accurately. In other words, for the red entries, we intentionally write wrong numbers as expected output.

In Figure 9, the $6^{\text {th }}$ input 00001275 means that the adder takes $(12)_{16}$ and (75) ${ }_{16}$ as input numbers to be added and the result is $(87)_{16}$, which is written as 00870000 as the two digits next to the most significant two digits represent the output of DUT. The $5^{\text {th }}$ entry is red because the addition result is different than the expected output, which is intentionally written false.

Similarly in Figure 10 , the $10^{\text {th }}$ input is 00000064 means that the input number is (64) ${ }_{16}$ and the actual square root result is 10 which is shown as $0000000 \mathrm{~A}$. It is shown as red because we intentionally write (003) ${ }_{16}$ as the expected output to check the proposed ATE.

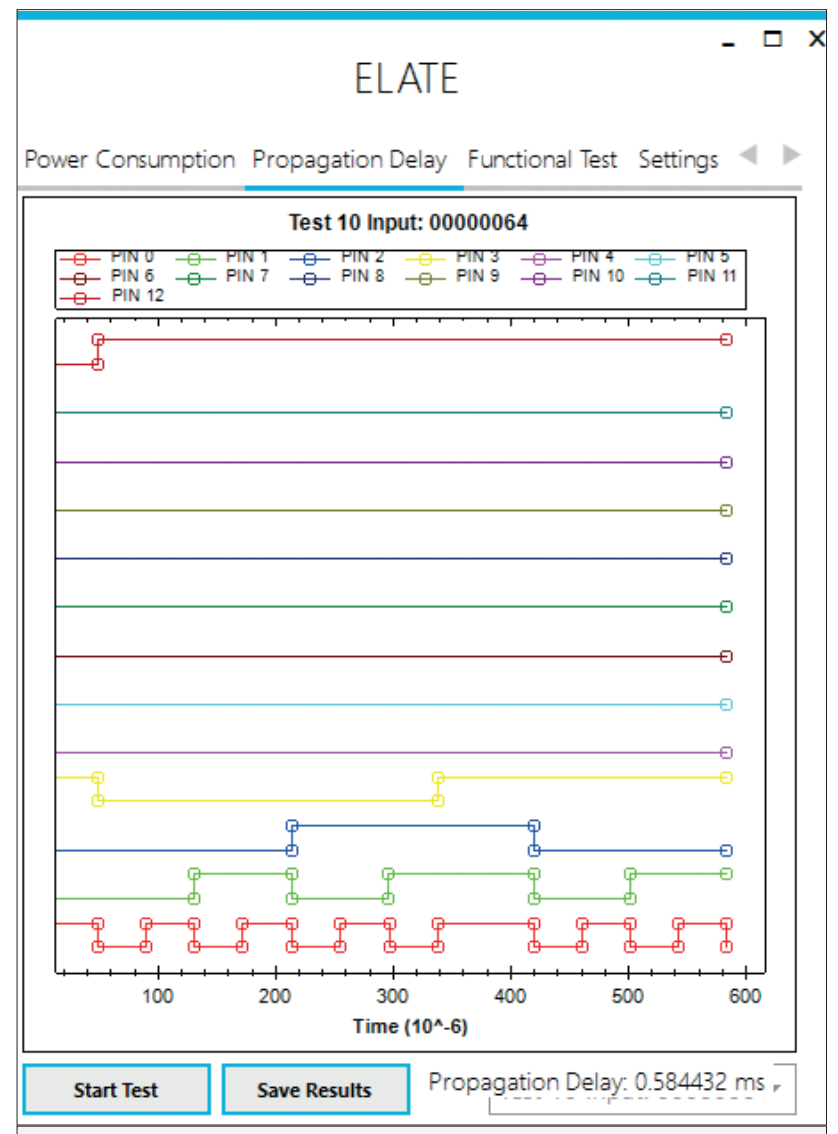

Figure 11. Propagation delay plot for input test vector $(64)_{16}$ 
Functional tests with 1024 random samples for each of all three test circuits resulted accurately.

\section{Propagation delay test results}

Propagation delay for square root test circuit is computed for 512 input test vectors increasing from 0 to $512^{2}$. Therefore the resultant square root results are $0,1, \ldots, 512$.

Figure 11 shows DUT outputs vs. time graph for a sample input test vector given to square root unit. It belongs to input 100 for which the resultant root is 10 as expected. As it is seen at the end

Table 2. Average and worst case delay for test circuits

\begin{tabular}{lcc}
\hline Circuit & Average Delay & Worst Case Delay \\
\hline 8 -bit Adder & $44.5 \mathrm{~ns}$ & $112 \mathrm{~ns}$ \\
\hline $12-$ bit adder & $49.5 \mathrm{~ns}$ & $84 \mathrm{~ns}$ \\
\hline
\end{tabular}

of the plot at Figure 11 the result for 100 (64 in hex) comes out to be 10, i.e. 00000000 1010. The worst case delay for the square root circuit is $29.8 \mathrm{~ms}$ whereas the average delay is $11.3 \mathrm{~ms}$ with increasing nature of delay by the increasing input value.

The propagation delay results for different test circuits are shown in Table 2. Average delay column shows the average delay for 1024 different test vectors generated randomly whereas the worst case delay column represents the worst delay among these 1024 test vectors.

The resultant worst case delay is higher for 8-bit adder although the average delay is smaller. This is because the 1024 input test vectors are selected randomly using srand() in C. But the average delay represents the characteristic better than the worst case one. Also in FPGAs, the delay depends on the usage of LUTs which may result in a convergence for the delays of 8-bit and 12-bit adders if same number of LUTs are utilized for both.

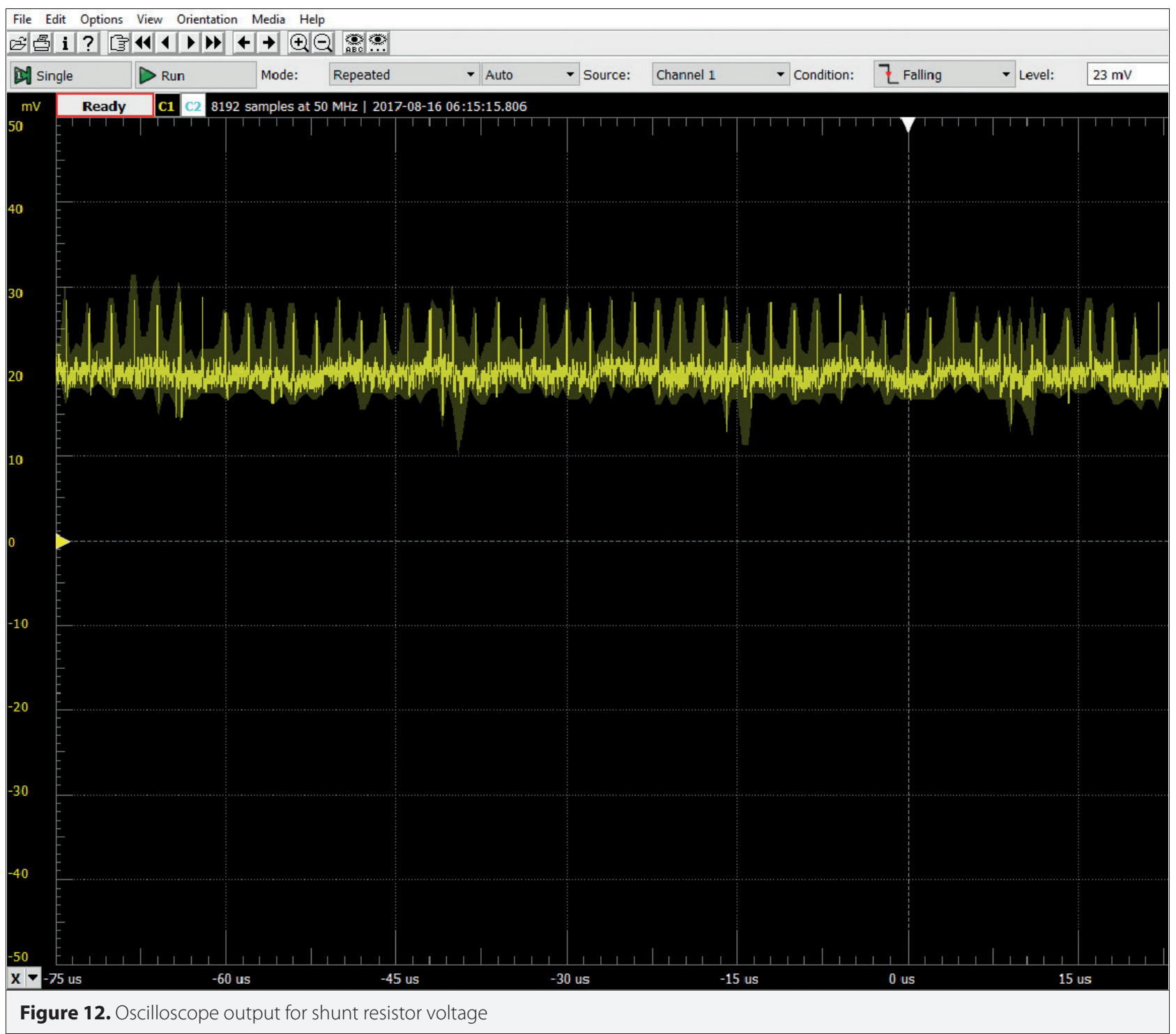




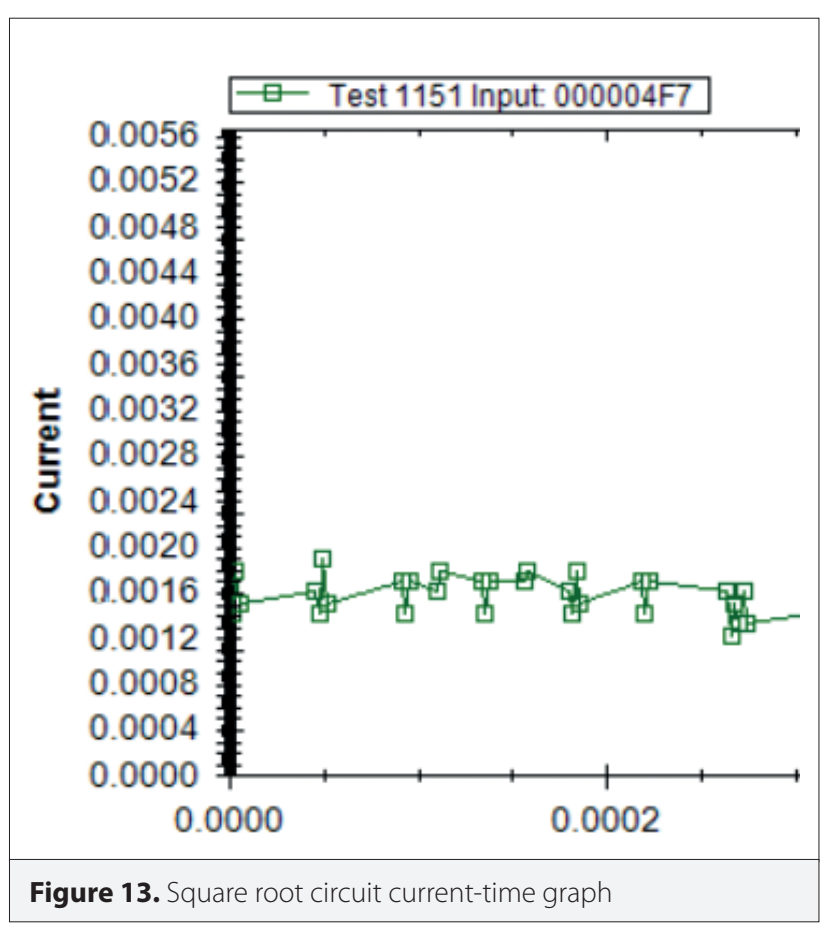

Power consumption test results

Power consumption is the weakest part of our ATE. Because the sampling rate of the used ADC is as low as $500 \mathrm{ksps}$, therefore the dynamic current fluctuations in nanosecond intervals cannot be captured by our ADC. However we tested the accuracy of our power consumption test by comparing with the Analog Discovery oscilloscope output where we only manage to measure the idle power consumption for the Stellaris Lauchpad MCU development board of Texas Instruments. Figure 12 shows the Analog Discovery oscilloscope output for shunt resistor voltage for 100 us interval and Figure 13 shows the resultant ATE shunt resistor current plot captured by ATE software for 300us interval. As the shunt resistor is about $110 \mathrm{hm}$ both plots give similar results which emprically verify the power consumption test result of ATE.

\section{Conclusion}

The proposed ATE performs very well in all functional tests. Although the final output voltage values are accurate for propagation delay voltage-time plots, further investigation and improvement are required in order to be sure about the actual delay. For that purpose precise tools to detect the actual de- lay of the circuits can be employed. Power consumption tests are working but the sampling rate of ADC must be really very high in tens of $\mathrm{GHz}$ in order to detect dynamic supply current changes. However, ATE proposed in this paper constitutes a good start from scratch and this model can further be improved by inserting more expensive but precise hardware as well as hardware and software enhancements.

Peer-review: Externally peer-reviewed.

Conflict of Interest: The author has no conflicts of interest to declare.

Financial Disclosure: This work is supported by The Scientific and Technological Research Council of Turkey (TUBITAK) under Grant No. 116 E296.

\section{References}

1. IC Test Systems - Advantest (March 2018).Available: https://www. advantest.com/products/ic-test-systems.

2. Production Board Test Teradyne (March 2018). Available: http:// www.teradyne.com/products/production-board-test.

3. Teseda Corporation: Silicon Failure Analysis and Yield Enhancement (March 2018). Available: http://www.teseda.com.

4. Teseda breaks $\$ 200$ per pin ATE barrier (March 2018). Available: http://www.eetimes.com/document.asp?doc_id=1175064.

5. L. Mostardini, L. Bacciarelli, L. Fanucci, L. Bertini, M. Tonarelli, M. De Marinis, "FPGA-based low-cost automatic test equipment for digital integrated circuits", IEEE International Workshop on Intelligent Data Acquisition and Advanced Computing Systems: Technology and Applications, Rende, pp. 32-37, 2009. [CrossRef]

6. J. H. M. Escobar, J. SachBe, S. Ostendorff, H. D. Wuttke, "Automatic generation of an FPGA based embedded test system for printed circuit board testing", $13^{\text {th }}$ Latin American Test Workshop (LATW), Quito, Ecuador, pp. 1-6, 2012. [CrossRef]

7. I. Aleksejev, A. Jutman, S. Devadze, S. Odintsov, T. Wenzel, "FPGA-based synthetic instrumentation for board test," IEEE International Test Conference, Anaheim, CA, pp. 1-10, 2012. [CrossRef]

8. A. A. Bayrakci, "ELATE: Embedded low cost automatic test equipment for FPGA based testing of digital circuits", 2017 10th International Conference on Electrical and Electronics Engineering (ELECO), Bursa, 2017, pp. 1281-1285.

9. F. S. Marques, R. P. Ribas, S. Sapatnekar, and A. I. Reis. "A new approach to the use of satisfiability in false path detection." Proceedings of the 15th ACM Great Lakes symposium on VLSI (GLSVLSI '05). ACM, New York, NY, USA, pp 308-311, 2005. [CrossRef]

10. N. Eén, N. Sörensson. "An extensible SAT-solver", International conference on theory and applications of satisfiability testing. Springer, Berlin, Heidelberg, 2003.

11. Documented Verilog UART (March 2018). Available: https://opencores.org/project/osdvu. 


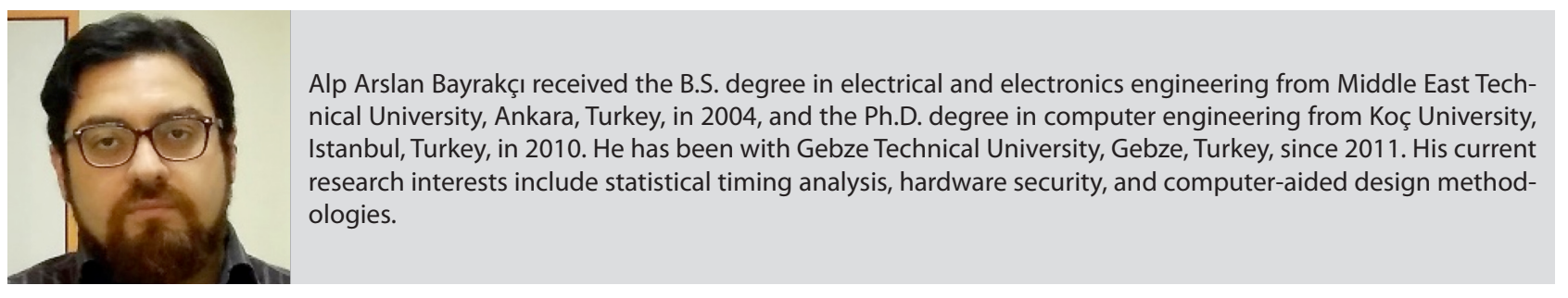

